\title{
The origin of X-ray emission of two distant $(z>1)$ cluster candidates with XMM-Newton
}

\author{
D. M. Neumann ${ }^{1}$, M. Arnaud ${ }^{1}$, C. Benoist ${ }^{2}$, L. da Costa ${ }^{3}$, H. E. Jørgensen ${ }^{4}$, L. F. Olsen ${ }^{4}$, \\ S. Bardelli ${ }^{5}$, E. Zucca ${ }^{5}$, S. Arnouts ${ }^{6}$, A. Biviano ${ }^{7}$, and M. Ramella ${ }^{7}$ \\ ${ }^{1}$ CEA/Saclay DSM/DAPNIA/SAp, L'Orme des Merisiers, 91191 Gif-sur-Yvette, France \\ 2 Observatoire de la Côte d'Azur, CERGA, BP 4229, 06304 Nice Cedex 4, France \\ ${ }^{3}$ ESO, Karl-Schwarzschild-Straße 2, 85748 Garching, Germany \\ 4 Astronomical Observatory, Juliane Maries Vej 30, 2100 Copenhagen, Denmark \\ 5 INAF - Osservatorio Astronomico di Bologna via Ranzani 1, 40127 Bologna, Italy \\ ${ }^{6}$ Laboratoire d'Astrophysique de Marseille, Traverse du Siphon - BP 8, 13376 Marseille Cedex 12, France \\ 7 INAF - Osservatorio Astronomico di Trieste via G.B. Tiepolo 11, 34131 Trieste, Italy
}

Received 18 March 2003 / Accepted 14 April 2003

\begin{abstract}
We present here a study of XMM-Newton data of two distant galaxy cluster candidates. One of these was discovered serendipitously in near infrared data, CL J0533-2411, the other one corresponds to the cluster EIS J0533-2412 part of the EIS cluster survey. The estimated redshift of CL J0533-2411 is $z=1.2-1.7$. EIS J0533-2412 is a rich system $\left(\Lambda_{\mathrm{cl}}=299\right)$, with a spectroscopically confirmed redshift of $z=1.3$. Both galaxy concentrations show firm X-ray detections, located within $30^{\prime \prime}$ of their optical center. However, we cannot resolve the sources with XMM-Newton. If the $\mathrm{X}$-ray emission originates from the $\mathrm{X}$-ray emitting intra-cluster medium (ICM) it would be extremely concentrated which is rather unlikely (core radii below $14 h_{65}^{-1} \mathrm{kpc}$ and $40 h_{65}^{-1} \mathrm{kpc}$, respectively). We argue that the X-ray sources are more likely AGN members of the galaxy concentrations. We set an upper limit for the bolometric luminosity of a hot ICM in the range $\sim 0.7-2.1 \times 10^{44} h_{65}^{-2} \mathrm{erg} / \mathrm{s}$ for CL J0533-2411, depending on the exact redshift. For EIS J0533-2412 the limit is $L_{\mathrm{bol}}=(6.2 \pm 1.4) \times 10^{43} h_{65}^{-2} \mathrm{erg} / \mathrm{s}$. We interpret our result in the following way: EIS J0533-2412 (and possibly CL J0533-2411) are proto-clusters and show matter overdensities before collapse, which explains the low significance of extended X-ray emission.
\end{abstract}

Key words. cosmology: observations - cosmology: large-scale structure of Universe - galaxies: clusters: individual: CL J0533-2411, EIS J0533-2412 - X-rays: galaxies: clusters - galaxies: active

\section{Introduction}

Cluster evolution with time provides essential information on the physics of structure formation and can set strong constraints on the cosmological parameters. In spite of remarkable progress in that field in recent years (e.g. Rosati et al. 2002 and references therein), the redshift range $z>1$ remains largely unexplored.

The results presented in this paper are part of an ongoing comprehensive effort to identify and study clusters at different epochs using as starting point the ESO Imaging Survey (EIS Nonino et al. 1999; Prandoni et al. 1999; Benoist et al. 1999) and the subsequent cluster candidate compilation, which was built using a matched filter technique in the $I$ band (Olsen et al. 1999; Scodeggio et al. 1999). These original data were complemented by multi-band optical/infrared imaging data to derive photometric redshifts. Combining these estimates with the

Send offprint requests to: D. M. Neumann,

e-mail: ddon@cea.fr positional information allows to search for concentrations in the 3D space and to select the most likely clusters (da Costa et al. 1999). First VLT spectroscopic observations confirmed the reality of three candidates in the redshift range $z=0.8-1.3$ (Benoist et al. 2002).

We present here XMM-Newton follow-up observations of two cluster candidates at $z>1$. The first object, CL J0533-2411, was detected serendipitously in the $J / K$ follow-up of EIS J0533-2412 with SOFI/NTT, as an overdensity in the projected distribution of galaxies with similar $J-K$ colors. Based on the $I, J$ and $K$-band data the estimated redshift of this cluster candidate is $z=1.2-1.7$. The other object is the system EIS J0533-2412, which is supposed to be a rich system based on the matched filter parameter $\Lambda_{\mathrm{cl}}$ $\left(\Lambda_{\mathrm{cl}}=299\right)$. VLT/FORS2 spectroscopic observations strongly suggest that this galaxy concentration is a true physical structure at $z=1.3$ (Benoist et al. 2002). The group in this field at $z=0.8$ as indicated by Benoist et al. (2002) is scattered through the field and does not coincide with the matched filter 
position of the EIS J0533-2412 cluster. Detailed spectral information of the cluster galaxies will be presented in a forthcoming paper by Jørgensen et al. (2003).

$\mathrm{X}$-ray observations are key to understanding the nature of these galaxy systems, which at such high redshift are not necessarily fully collapsed and relaxed objects. They could be filaments, proto-clusters or more or less virialised clusters. The detection of extended X-ray emission from hot gas, heated by gravitational collapse, would trace the virialized portion of these distant and young systems.

The paper is structured in the following way. The data are described in Sect. 2. In Sect. 3 we present the data reduction and the results of our analysis. We examine the significance and extent of the X-ray emission at the location of both cluster candidates, the physical origin of the emission and put constraints on the ICM X-ray luminosities. We discuss the physical nature of the cluster candidates in Sect. 4 and conclude in Sect. 5.

Throughout this paper we use $H_{0}=65 h_{65} \mathrm{~km} \mathrm{~s}^{-1} \mathrm{Mpc}^{-1}$, and $\Omega_{\mathrm{m}}=0.3, \Omega_{\Lambda}=0.7$. In this cosmology, one arcminute corresponds to $0.541 \mathrm{Mpc}$ and $0.547 \mathrm{Mpc}$ at a redshift of $z=$ 1.3 and $z=1.7$, respectively.

\section{The XMM-Newton data}

Both clusters lie sufficiently close together ( 2 arcmin distance) to be observed in one XMM-Newton observation. The data were taken in two exposures: $64.9 \mathrm{ksec}$ during revolution 332 and $34.5 \mathrm{ksec}$ during revolution 335 . The total exposure time was longer than originally scheduled since the pn-camera was not operational at that time. Thus we present here only data based on EPIC-MOS cameras.

\section{Data analysis}

For our analysis we use the official XMM-Newton software (SAS version 5.2). The data presented below are corrected for vignetting effects using the photon weighting method (Arnaud et al. 2001; Majerowicz et al. 2002).

\subsection{Background screening}

XMM-Newton observations suffer from time periods of high background due to soft protons from solar flares. During those flares the background level can increase by several orders of magnitude as compared to the quiescent background level and significantly degrades the signal to noise ratio.

We bin the MOS-data in time intervals of $100 \mathrm{~s}$ in the high energy $10-12 \mathrm{keV}$ band, where the emission is dominated by the particle-induced background. We screen the data for flare periods by applying a rejection threshold in this energy band of $>15$ counts. After such cleaning, the effective exposure time is $83 \mathrm{ksec}$ and $84 \mathrm{ksec}$ for the MOS1 and MOS2 cameras, respectively.

\subsection{Source detection and morphology}

In order to enhance the signal-to-noise ratio we only take into account data in the energy band $0.3-3.0 \mathrm{keV}$. We
Table 1. Object position.

\begin{tabular}{lll}
\hline \hline Objects & RA $(\mathrm{J} 2000.0)$ & Dec $(\mathrm{J} 2000.0)$ \\
\hline CL J0533-2411 & $05^{\mathrm{h}} 33^{\mathrm{m}} 44.0^{\mathrm{s}}$ & $-24^{\circ} 11^{\prime} 01^{\prime \prime}$ \\
XMMU J053344.4-241048 & $05^{\mathrm{h}} 33^{\mathrm{m}} 44.4^{\mathrm{s}}$ & $-24^{\circ} 10^{\prime} 48^{\prime \prime}$ \\
\hline EIS J0533-2412 & $05^{\mathrm{h}} 33^{\mathrm{m}} 39.2^{\mathrm{s}}$ & $-24^{\circ} 12^{\prime} 58^{\prime \prime}$ \\
$R$ clump & $05^{\mathrm{h}} 33^{\mathrm{m}} 38.0^{\mathrm{s}}$ & $-24^{\circ} 12^{\prime} 59^{\prime \prime}$ \\
XMMU J053337.8-241250 & $05^{\mathrm{h}} 33^{\mathrm{m}} 37.8^{\mathrm{s}}$ & $-24^{\circ} 12^{\prime} 50^{\prime \prime}$ \\
\hline
\end{tabular}

extract the MOS1\&2 image with a pixel size of $1.1^{\prime \prime}$ times $1.1^{\prime \prime}$, which corresponds to the physical size of the detector pixels. The X-ray signal at the location of both CL J0533-2411 and EIS J0533-2412 appear very faint and we therefore apply first a detection algorithm, which gives the significance of the signals. We use the code described in Neumann \& Böhringer (1997), which assumes Poisson statistics and uses a Gaussian filter. We apply this algorithm with a Gaussian filter size of $\sigma=5.5^{\prime \prime}$ and an overall background estimate of $0.004 \mathrm{cts} / \mathrm{s} / \mathrm{arcmin}^{2}$ in the $0.3-3.0 \mathrm{keV}$ energy range.

Figure 1 shows the X-ray contours in sigma over the $K$-band image obtained with SOFI/NTT. Figure 2 shows a zoom on EIS J0533-2412, where the X-ray contours are overlaid on the $R$-band VLT/FORS2 image. The position of the galaxies with same $J-K(J-K=2-2.4)$ color are indicated by squares and the three EIS J0533-2412 galaxies with measured redshifts by circles.

Significant X-ray emission is detected at both cluster locations. The peak positions are given in Table 1, together with the optical center of the cluster candidates estimated from a $3 \mathrm{D}$ analysis of the galaxy concentrations using the IJK imagery data. A $6 \sigma$ compact X-ray source (XMMU J053344.4-241048) is detected only $15^{\prime \prime}$ away from the optical center of CL J0533-2411. For EIS J0533-2412, a possibly more extended X-ray source (XMMU J053337.8-241250) is detected at the $5 \sigma$ level, with a faint extension in the North-West direction. However this extension coincides with bright foreground galaxies and might be due to contamination. Roughly 40 arcsec east of XMMU J053337.8-241250 there is another $3 \sigma$ detection (indicated with an "S" in Fig. 1). The estimated optical center of EIS J0533-2412 is located in between these two X-ray peaks, with an offset with respect to the XMMU J053337.8-241250 peak of 29". However, the deeper $R$-band VLT image reveals a concentration of faint galaxies (hereafter called the " $R$ clump"), centered on the galaxy \#3 at $z=1.298$ (right circle in Fig. 1 and Table 1), only $10^{\prime \prime}$ from the $\mathrm{X}$-ray peak with $5 \sigma$ detection.

\subsection{Source extent}

In order to determine the origin of the X-ray emission, we examine in the following the extent of XMMU J053344.4-241048 and XMMU J053337.8-241250 by analyzing their surface brightness profiles. We remove all sources detected above $3 \sigma$ in the extraction region aperture, in particular the secondary X-ray source found east of XMMU J053337.8-241250 (indicated with an "S" in Fig. 1). 


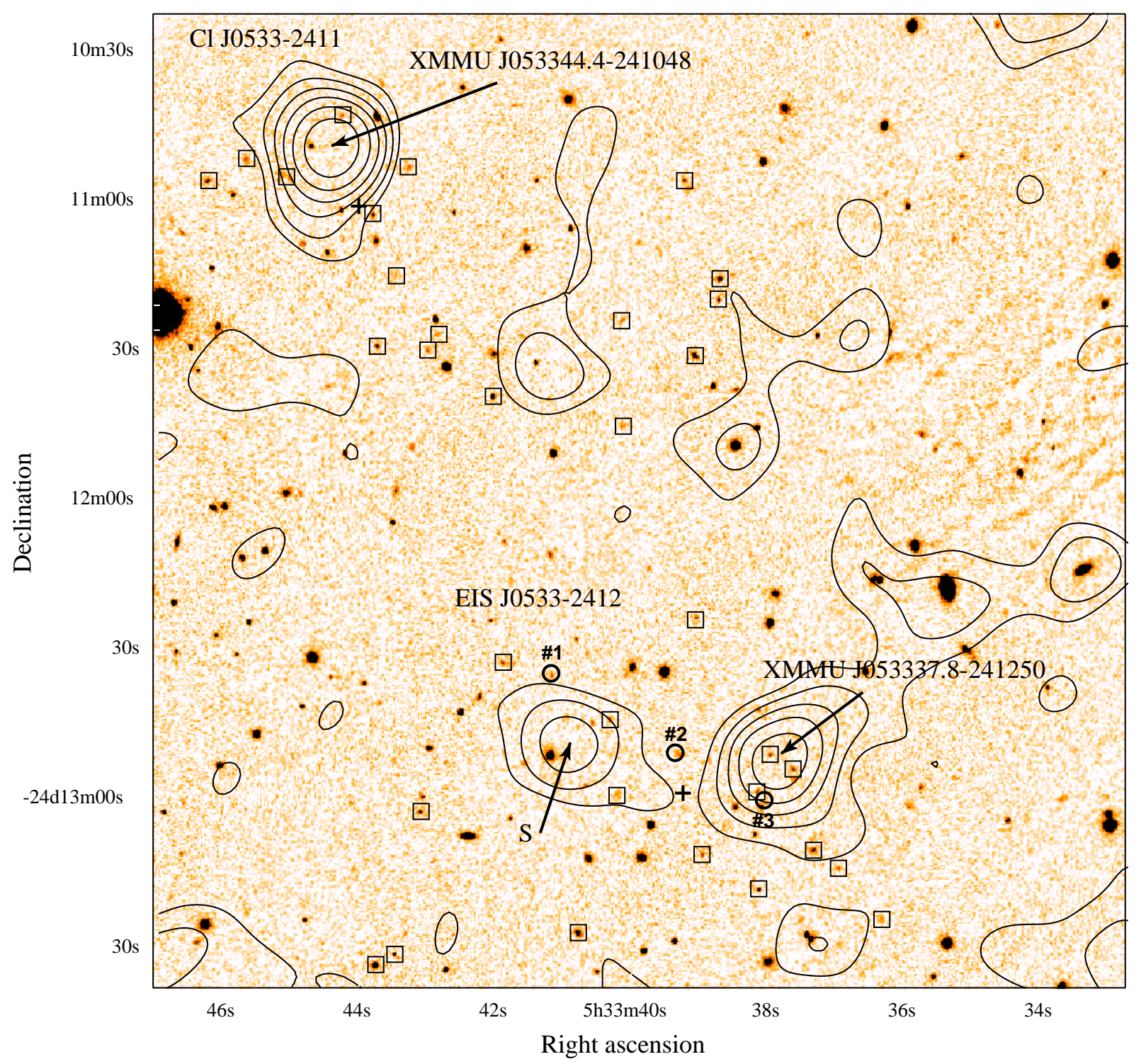

Fig. 1. The contours of the X-ray emission in sigmas of significance. Lowest contour and stepwidth 1 sigma. The contours are overlaid over the SOFI/NTT image taken in the $K$-band. The squares indicate galaxies with $J-K=2-2.4$ corresponding to early type galaxies with redshifts approximately beyond 1.0. The three spectroscopically confirmed members of EIS J0533-2412 are indicated by circles. The crosses show the center of the galaxy concentrations.

\subsubsection{XMMU J053344.4-241048}

Figure 3 shows the azimuthally averaged MOS1\&2 surface brightness profile of XMMU J053344.4-241048. The center was fixed at the peak of the X-ray emission (Table 1). To obtain the merged surface brightness profile of the source we extract first the surface brightness profile for each exposure (performed in revolution 332 and revolution 335) and each camera (MOS1 and MOS2). Then we merge the surface brightness profiles of the two exposures for each camera individually together and finally add the profiles of the two cameras together.

In order to check whether this source is extended we compared the surface brightness profile with the PSF model (Ghizzardi 2001) and adding a flat background. The results are shown in Fig. 3. The derived $\chi^{2}$ is 36.5 for 34 degrees of freedom (d.o.f.) indicating that the emission is consistent with that of a point source. Fitting a $\beta$-model instead (with $\beta=2 / 3$ ) convolved with the PSF does not improve the quality of the fit. The best fit is actually obtained for a point-like source emission $\left(\Theta_{\mathrm{c}}=0\right)$. We derived an upper limit for the core radius of $\Theta_{c}=0.026^{\prime}$ at the $99 \%$ confidence level $\left(\Delta \chi^{2}=6.635\right)$. The corresponding profile is plotted as a dotted line in Fig. 3. 0.026' corresponds to roughly $14 h_{65}^{-1} \mathrm{kpc}$ at $z=1.3-1.7$.

\subsubsection{XMMU J053337.8-241250}

Defining the center of XMMU J053337.8-241250 is more complicated since the source seems a priori to be more extended than XMMU J053344.4-241048 and somewhat fainter (see Fig. 1). The determination of the center is very important since a wrong choice could lead to an artificial measured extent when compared to a point-like source. 


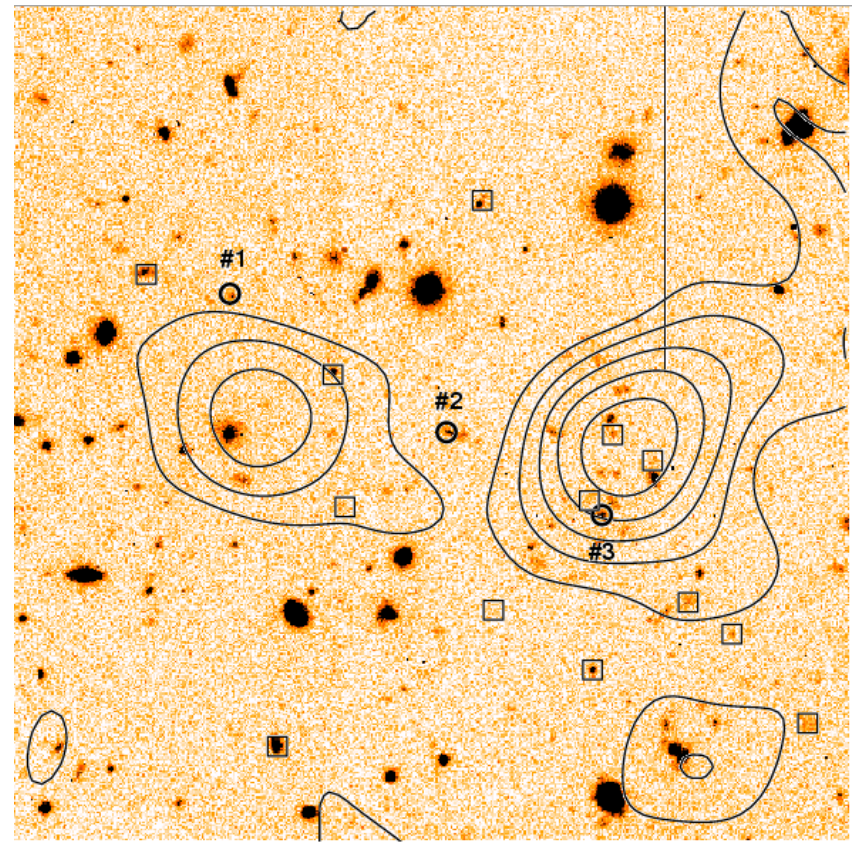

Fig. 2. Zoom on EIS J0533-2412. The contours of the X-ray emission are overlaid over the VLT/FORS2 image taken in $R$-band. The exposure of the VLT-image is $10 \mathrm{~min}$. Same symbols as in Fig. 1.

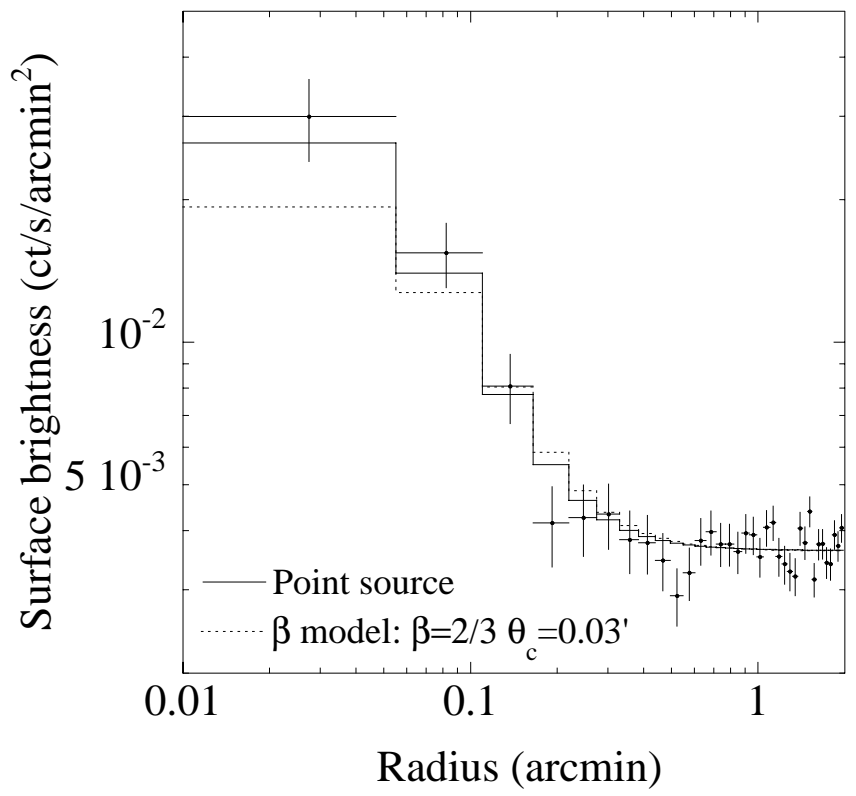

Fig. 3. Surface brightness profile of XMMU J053344.4-241048. The profile is summed over MOS1 and MOS2. The error bars for the surface brightness are $1 \sigma$ uncertainties. The full line corresponds to the point source model by Ghizzardi (2001) plus background. The dotted line is a beta-model convolved with the PSF of XMM-Newton with $\beta=2 / 3$ and $\Theta_{c}=0.026^{\prime}$. This model is marginally consistent with the data (see text).

We first extracted the profile centered at the peak of the whole emission. We also consider a more conservative approach, which will maximize the profile concentration. For each exposure (in total two) and each camera (MOS1 and MOS2), we further extracted four profiles around the X-ray

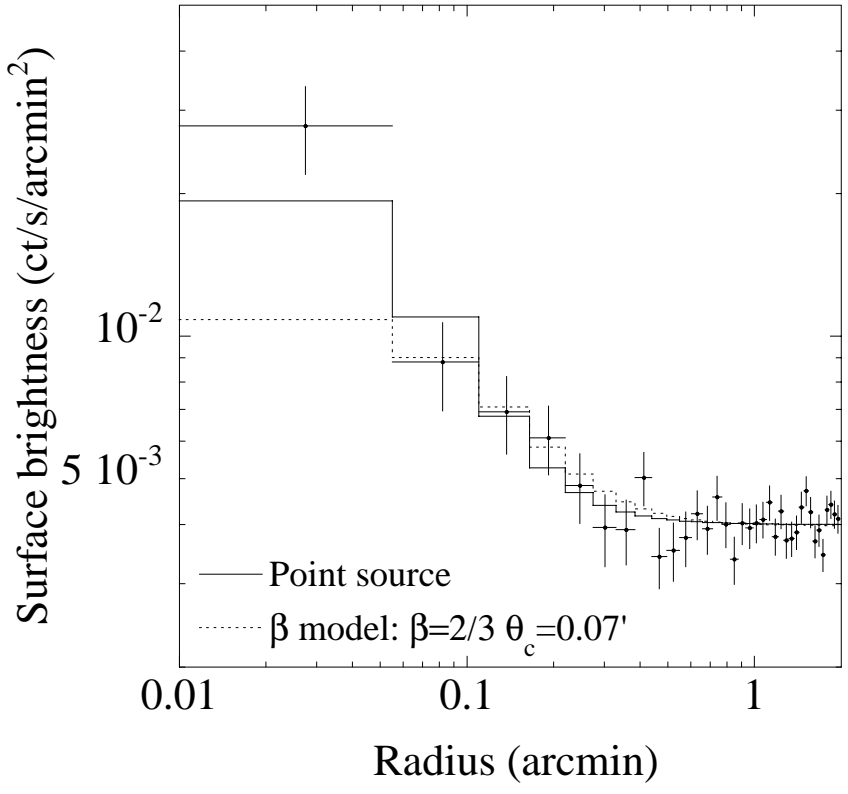

Fig. 4. Surface brightness profile of XMMU J053337.8-241250. The profile is summed over MOS1 and MOS2. Error bars and full line are identical to Fig. 3. The dotted line is a beta-model convolved with the PSF of XMM-Newton with $\beta=2 / 3$ and $\Theta_{c}=0.073^{\prime}$. This model is marginally consistent with the data (see text).

peak. The centers of these profiles span a quadrant of $2 \times$ 2 arcsec with the X-ray peak in its center. We choose this spacing since a small offset between center source and measured center will not be visible within 2 arcsec, the FWHM of the XMM-Newton mirrors being in the order of 4-6 arcsec. Among the 5 resulting surface brightness profiles per exposure and camera we choose the profile with the highest central intensity and then merge the different brightness profiles together. The derived profile is consistent with the profile centered at the overall X-ray peak but is more noisy. Since the two profiles are consistent, we consider in the following only the latter profile extracted using the overall center.

The surface brightness profile together with the best fit surface brightness profile of a point source model including background is shown in Fig. 4. Again a good fit is obtained with a derived $\chi^{2}$ of 34.3 for 34 d.o.f. Fitting a $\beta$-model convolved with the PSF to the brightness profile gives again a best fit for a point source. The $99 \%$ confidence level for $\Theta_{c}$ in this case is $\Theta_{\mathrm{c}}<0.073^{\prime}$ for $\beta=2 / 3$. This corresponds to $40 h_{65}^{-1} \mathrm{kpc}$ at $z=1.3$.

\subsection{Count rate estimates}

The total MOS1\&2 count rate was estimated by integrating the surface brightness profiles, after subtracting the best fit background level. The integration radius was chosen to be $20^{\prime \prime}$ to optimize the $\mathrm{S} / \mathrm{N}$ ratio. However, this is smaller than the size of the PSF and we have to correct for the flux loss outside the integration radius. Since the sources are not resolved, the correction factor was estimated from the Encircled Energy Fraction within $20^{\prime \prime}$ for the PSF model (EEF 74\%). For XMMU J053344.4-241048 (close to CL J0533-2411) we derived a PSF corrected count rate in the $0.3-3 \mathrm{keV}$ 
energy band of $(1.3 \pm 0.2) \times 10^{-3} \mathrm{ct} / \mathrm{s}$. The count rate for XMMU J053337.8-241250 (close to EIS J0533-2412) is (9.6 \pm 2.1) $\times 10^{-4} \mathrm{ct} / \mathrm{s}$.

\subsection{The origin of the $X$-ray emission}

The XMM observations of both CL J0533-2411 and EIS J0533-2412 clearly reveal X-ray emission. However, the extracted surface brightness profiles of the X-ray sources are comparable to the shape of the PSF and are therefore not resolved. Consequently, we cannot unambiguously determine the physical origin of the sources, especially since little is known about X-ray clusters beyond $z=1$.

It is, however, instructive to compare the upper limit set on the source extent $\left(r_{\mathrm{c}}<14 h_{65}^{-1} \mathrm{kpc}\right.$ and $40 h_{65}^{-1} \mathrm{kpc}$ at the $99 \%$ confidence limit) with the properties of the four $z>1$ clusters known with extended X-ray emission. The two RDCS clusters (Rosati et al. 1998) with relatively regular morphology, RX J0848.9+4452 ( $z=1.27)$ and RX J0910+5422 ( $z=1.1)$, have core radii at least twice as large: $100 \pm 30 h_{65}^{-1} \mathrm{kpc}$ (Stanford et al. 2001) and $171 \pm 53 h_{65}^{-1} \mathrm{kpc}$ (Stanford et al. 2002). Interestingly, the other RDCS cluster, CIG J0848+4453 $(z=1.26)$, was originally discovered via a search for clusters in infrared bands (Stanford et al. 1997). This cluster has an irregular morphology (Stanford et al. 2001). Nevertheless, a $\beta$-model fit gives a core radius of $\sim 200 h_{65}^{-1} \mathrm{kpc}$ (Holden et al. 2002). A cluster with a core radius of this size at this redshift is resolved by XMM. Finally, RX J1053.7+5735 $(z=1.26)$, discovered in deep ROSAT observations in the Lockman hole (Hasinger et al. 1998), was clearly resolved by XMM as a bimodal cluster (Hashimoto et al. 2002).

A comparison can also be made with the expectation of the self-similar model of cluster formation, where $r_{\mathrm{c}}$ scales as $r_{\mathrm{c}} \propto r_{200} \propto h(z)^{-1}$ (e.g. Evrard \& Gioia 2002). The typical core radius of nearby clusters is $r_{\mathrm{c}} \sim 0.12 r_{200}$ (Neumann $\&$ Arnaud 1999) where $r_{200}=2.84(T / 10 \mathrm{keV})^{1 / 2} h_{65}^{-1} \mathrm{Mpc}$ (Evrard et al. 1996). For a relaxed part of a distant cluster with a temperature as low as $2 \mathrm{keV}$, we would thus expect a core radius of $73 h_{65}^{-1} \mathrm{kpc}(z=1.3)$ and $59 h_{65}^{-1} \mathrm{kpc}(z=1.7)$. These core radii are again excluded by our data.

In conclusion, although we cannot totally exclude that the $\mathrm{X}$-ray emission is due to exceptionally concentrated ICM, the upper limit found on the source extent clearly favors a point source (AGN) origin. Those AGNs can be either foreground/background galaxies, or members of the galaxy overdensity.

The latter hypothesis is a priori more likely in view of the source locations, remarkably close to the optical center of the galaxy concentrations, CL J0533-2411 and EIS J0533-2412, respectively. We further quantify this point. Assuming a power law AGN emission with a photon index of $\Gamma=2$, the measured count rate of XMMU J053344.4-241048 and XMMU J053337.8-241250 corresponds to an unabsorbed flux in the [0.5-2] keV energy band of $S_{1}=3.3 \times 10^{-15} \mathrm{erg} / \mathrm{s} / \mathrm{cm}^{2}$ and $S_{2}=2.4 \times 10^{-15} \mathrm{erg} / \mathrm{s} / \mathrm{cm}^{2}$, respectively. From the deep $X M M-N e w t o n$ survey of the Lockman Hole region (Hasinger et al. 2001), the number of sources (essentially AGN) per square degrees above those flux limits are $N\left(>S_{1}\right) \sim 300$ and $N\left(>S_{2}\right) \sim 400$. The probability that a serendipitous AGN at least as bright as XMMU J053344.4-241048 is found by chance within $15^{\prime \prime}$ of the center of CL J0533-2411 is only 1.6\%. For XMMU J053337.8-241250 (located at 10" from the center of the R clump in EIS J0533-2412), the probability is even smaller: $1.1 \%$. If we consider a conservative distance of $30^{\prime \prime}$ (about $270 \mathrm{kpc}$ ) to take into account possible uncertainties on the optical center determination, these probabilities are still below $10 \%$.

We looked for possible optical counterparts, as well as radio sources in the field. For that purpose, we used the NRAO VLA Sky Survey, NVSS (Condon et al. 1998). Within $30^{\prime \prime}$ in radius of the optical center of both concentrations, we only found one NVSS source, NVSS J053338-241301. This source is located only $2^{\prime \prime}$ away from the galaxy member \#3 of EIS J0533-2412, an emission line galaxy, and is probably associated with it. However, it is too far away from XMMU J053337.8-241250 (10") to be its counterpart, the positional uncertainty of EPIC/MOS being less than $5^{\prime \prime}$. It could however contaminate the X-ray emission. On the other hand there are at least two photometric members (see Fig. 1) within the error box of the X-ray source, which could be associated with it. For XMMU J053344.4-241048 there is a bright optical source at a distance of $5^{\prime \prime}$ of the maximum of the X-ray emission but this source is not part of the colour selected galaxies. However, there are several faint $R$ galaxies around the X-ray maximum but with no photometric redshift information.

\subsection{Upper limit on the ICM luminosity}

Since, as discussed above, it is very likely that the X-ray emission we observe is at least heavily contaminated by AGN emission, the source count rates must be regarded as upper limits for a non-resolved ICM. However, there might still be ICM emission more diffuse than the detected X-ray sources and hidden within the background. We estimate an upper limit (at the $3 \sigma$ level) on this emission by fitting the profiles with a point source plus a beta model with a core radius of $100 h_{65}^{-1} \mathrm{kpc}$ (see above) and $\beta=2 / 3$. We obtained an upper limit of $\sim 6.2 \times 10^{-4} \mathrm{ct} / \mathrm{s}$, lower than the count rates of the X-ray sources. Since these count rates are lower than the actual measured source count rates, the observed count rates can thus be considered as firm upper limits on the overall ICM emission.

From the measured source count rates we derive an upper limit on the ICM luminosities of the two cluster candidates. To convert the actual count rate, $(1.3 \pm 0.2) \times 10^{-3} \mathrm{ct} / \mathrm{s}$ for XMMU J053344.4-241048 and $(9.6 \pm 2.1) \times 10^{-4} \mathrm{ct} / \mathrm{s}$ for XMMU J053337.8-241250, to luminosity we used the XSPEC package and the EPIC/MOS response. We use an absorbed MEKAL model with an assumed temperature $k T=2 \mathrm{keV}$, a hydrogen column density of $N_{\mathrm{H}}=2.4 \times 10^{20} \mathrm{~cm}^{-2}$ (Dickey \& Lockman 1990), and a metal abundance of 0.3 times the solar value. For CL J0533-2411 we do not have a precise redshift estimate. The derived bolometric X-ray luminosities are $L_{\text {bol }}=(1.8 \pm 0.3) \times 10^{44} h_{65}^{-2} \mathrm{erg} / \mathrm{s}$ for $z=1.7$ and 
$L_{\text {bol }}=(8.4 \pm 1.3) \times 10^{43} h_{65}^{-2} \mathrm{erg} / \mathrm{s}$ for $z=1.3$. For EIS J0533-2412 we derived $L_{\text {bol }}=(6.2 \pm 1.4) \times 10^{43} h_{65}^{-2} \mathrm{erg} / \mathrm{s}$.

\section{Discussion}

\subsection{The nature of the cluster candidates}

The upper limit on the ICM bolometric luminosity is low enough for EIS J0533-2412 to shed some light on the nature of this galaxy system. We recall that spectroscopic VLT observations indicate that this is a real physical system, and not just a chance alignment of galaxies. First we note that the upper limit on the luminosity of EIS J0533-2412 is similar to the luminosity of a poor cluster like Virgo in the nearby Universe, $\sim 4 \times 10^{43} h_{65}^{-2} \mathrm{erg} / \mathrm{s}$ (Arnaud \& Evrard 1999). On the other hand, the matched filter parameter, $\Lambda_{\mathrm{cl}}$, an estimate of the effective optical luminosity, is especially high: $\Lambda_{\mathrm{cl}}=299$. Using at face value the correlation ${ }^{1}$ between $\mathrm{X}$-ray bolometric luminosity and $\Lambda_{\mathrm{cl}}$ derived by Donahue et al. (2001) from their joint optical/ROSAT survey, we would expect a bolometric luminosity of at least $3 \times 10^{45} h_{65}^{-2} \mathrm{erg} / \mathrm{s}$. This is twice the luminosity of Coma, $\sim 1.4 \times 10^{45} h_{65}^{-2} \mathrm{erg} / \mathrm{s}$ (Arnaud \& Evrard 1999). This apparent contradiction can be naturally alleviated if we simply assume that EIS J0533-2412 is a massive proto-cluster. In other words, EIS J0533-2412 is a rich overdensity of galaxies (as indicated by the high $\Lambda_{\mathrm{cl}}$ value), which is still largely in a previrialised state (hence a low X-ray luminosity because the gravitational potential is not deep enough to heat all the gas at the virial temperature). In view of the galaxy distribution, we can even speculate further. The probable structure members (selected on their $J-K$ color) seem to follow a $\mathrm{S}-\mathrm{W} / \mathrm{N}-\mathrm{E}$ filamentary structure, with the $R$ clump in the middle. This $R$ clump could be the "seed" (may be partially virialized) onto which the whole structure will finally collapse. To further check this hypothesis we need more spectroscopic VLT measurements to fully assess the 3D structure of the galaxy concentration and the dynamical state of the $R$ clump.

Since we have neither spectroscopic redshift measurement nor richness information on CL J0533-2411, the nature of this galaxy concentration remains entirely open. We can only tell it is not a very massive cluster in view of the upper limit on the $\mathrm{X}$-ray luminosity.

We found evidence of AGN activity in the center of both galaxy concentrations. Interestingly, there is also evidence of such activity in RX J0910+5422 $(z=1.1)$, a massive cluster in a much more advanced stage of formation. High resolution Chandra deep observation indeed revealed the presence of 3 AGNs associated with cluster galaxies within the central $\theta<30^{\prime \prime}$ part of the cluster. This aparently common AGN activity in high $z$ (proto) clusters might simply reflect a high AGN activity in the past: the luminosity function of X-ray selected AGNs shows a strong density evolution at redshift up to $\sim 1.5$ (Miyaji et al. 2000). AGN activity could also be further boosted in regions of high galaxy concentration as compared to the field (environmental effects). To assess this issue requires

\footnotetext{
${ }^{1} \log \left(L_{\mathrm{bol}, 44}\right)=(-6.9 \pm 1.1)+(3.6 \pm 0.8) \log \left(\Lambda_{\mathrm{cl}}\right)$ for $\Omega_{\mathrm{m}}=1$ and $H_{0}=75 \mathrm{~km} \mathrm{~s}^{-1} \mathrm{Mpc}^{-1}$. For the cosmological parameters considered here, the luminosity must be multiplied by 2.47 at $z=1.3$.
}

statistical analysis of combined Large Scale X-ray/optical surveys (e.g. the XMM-LSS, Pierre et al. 2003). Inversely, if AGN activity for galaxies is indeed higher in overdense regions then looking at the surroundings of AGN might be an interesting way to find distant galaxy clusters.

\subsection{Searching for virialized massive high redshift clusters}

Calculating the ratio of number density of massive high to low redshift clusters is a powerful tool for the determination of cosmological parameters (Perrenod 1980). The higher this ratio, the lower the corresponding density parameter $\Omega_{\mathrm{m}}$. Thus, in principle it is sufficient to detect distant massive clusters and to compare their number density to nearby ones to determine $\Omega_{\mathrm{m}}$. However, despite the launch of XMM-Newton and Chandra the detection of extended X-ray emission of clusters of galaxies at redshifts beyond unity is still very difficult. As already mentioned, only four clusters with $z>1$ with extended X-ray emission have been found so far, and their corresponding X-ray luminosities lie well below $L_{\text {bol }}=10^{45} \mathrm{erg} / \mathrm{s}$, which makes them not very massive objects. Up to now those clusters were either found in deep X-ray observations (Hasinger et al. 1998; Rosati et al. 1999), or via a search for clusters in infrared bands (Stanford et al. 1997). These detection techniques require relatively long exposure times and/or the use of the largest telescopes available with small field-of-view. This causes the sky coverage to be very small, which makes it difficult to assess the number density of massive distant clusters with high precision and which is furthermore biased by the various detection techniques applied.

Our approach for detecting massive galaxy clusters via a search in optical/infrared bands and the lack of finding clear evidence for ICM emission underlines the above mentioned difficulty. Recent studies indicate that there is clearly a decoupling of optical and X-ray parameters for clusters at redshifts above unity (Donahue et al. 2002). There are a lot of promising distant cluster candidates detected with high signal in optical wavelengths, which at first sight, surprisingly, do not have a corresponding luminous X-ray counterpart. At redshifts below unity, the correlation between optical and X-ray luminosity seem to match better, as was indicated recently by Donahue et al. $(2001,2002)$.

What could be a possible explanation for this? One answer could be that the optical/infrared indicators do not match galaxies embedded in clusters at $z>1$. This implies a notyet-accounted-for evolution of galaxy properties. Another explanation could be that there is no or not substantial evolution in the galaxy physics of clusters but strong evolution of their $\mathrm{X}$-ray properties and dark matter halos around $z=1$. This could imply that massive clusters and their corresponding dark matter halos that we observe today formed or collapsed at redshifts close to 1 . If this is true, distant clusters detected in $\mathrm{X}$-rays at redshifts close to unity should show a high degree of significant substructure. In fact, all clusters clearly detected in X-rays at these redshifts show indications of substantial substructure, which strengthens the hypothesis of important cluster 
evolution at $z=1$. In this case galaxy overdensities found in optical/infrared wavelength bands, such as the two cluster candidates presented here, could show the overdensities or cluster "seeds", which then collapse later into clusters detectable as extended X-ray sources. The galaxy density enhancements can in this case be interpreted as a tracer of regions of future cluster formation.

\section{Conclusion}

The XMM-Newton observations of two promising high redshift $(z>1)$ cluster candidates found in optical/infrared wavelength bands, CL J0533-2411 $(z=1.2-1.7)$ and EIS J0533-2412 $(z=1.3)$, reveal clear X-ray emission. However, the extracted surface brightness profiles of the two corresponding X-ray sources, XMMU J053344.4-241048 and XMMU J053337.8-241250, are comparable with the shape of the PSF. We argue that the X-ray sources are very likely AGN members belonging to the galaxy concentrations.

We derived an upper limit for the bolometric luminosity of a hot ICM in the range $\sim 0.7-2.1 \times 10^{44} h_{65}^{-2} \mathrm{erg} / \mathrm{s}$ for CL J0533-2411, depending on the exact redshift. For EIS J0533-2412 the limit is $L_{\text {bol }}=(6.2 \pm 1.4) \times 10^{43} h_{65}^{-2} \mathrm{erg} / \mathrm{s}$. This low luminosity found for a rich spectroscopically confirmed galaxy system can be explained if EIS J0533-2412 is in fact a massive proto-cluster which has not yet had sufficient time to collapse.

Our results indicate that rich galaxy overdensities at high redshift found in optical/infrared surveys are not necessarily massive virialised clusters. On the other hand they are of unique value for our understanding of structure formation and the dynamics of cluster collapse. Such surveys can reveal previrialised large galaxy overdensities or cluster "seeds", which will collapse later into clusters detectable as extended X-ray sources. Detailed multiwavelength follow-up of large optical surveys is essential. Multi-object spectroscopy is required not only to confirm the physical reality of the systems, but also to fully address their 3D structure and the dynamical state of the structure. Combined observations with high resolution X-ray instruments (like Chandra) and high throughput instruments (like XMM-Newton) are needed at the same time to i) assess the level of AGN activity which might be high in such young systems ii) map the virialised gaseous part of the structures.

Acknowledgements. We would like to thank S. Majerowicz for providing the code to extract surface brightness profiles from photonevent tables from XMM-Newton data.

\section{References}

Arnaud, M., \& Evrard, A. 1999, MNRAS, 305, 631

Arnaud, M., Aghanim, N., Gastaud, R., et al. 2001, A\&A, 365, L67

Benoist, C., da Costa, L., Jørgensen, H. E., et al. 2002, A\&A, 394, 1

Benoist, C., da Costa, L., Olsen, L. F., et al. 1999, A\&A, 346, 58

Condon, J. J., Cotton, W. D., Greisen, E. W., et al. 1998, AJ, 115, 1693.

da Costa, L., Scodeggio, M., Olsen, L. F., et al. 1999, A\&A, 343, L29

Dickey, J. M., \& Lockman, F. J. 1990, ARA\&A, 28, 215

Donahue, M., Mack, J., Scharf, C. A., et al. 2001, ApJ, 552, L93

Donahue, M., Scharf, C. A., Mack, J., et al. 2002, ApJ, 569, 689

Evrard, A. E., Metzler, C. A., \& Navarro, J. F. 1996, ApJ, 469, 494

Evrard, A. E., \& Gioia, I. 2002, Merging Processes in Galaxy Clusters, ed. L. Feretti, I. M. Gioia, \& G. Giovannini, Astrophys. Space Sci. Library, 272, 253

Ghizzardi, S. 2001, In Flight Calibration of the PSF for the MOS1 and MOS2 Cameras, XMM-SOC-CAL-TN-0022, http://xmm.vilspa.esa.es/user/calib_top.html

Hashimoto, Y., Hasinger, G., Arnaud, M., Rosati, P., \& Miyaji, T. 2002, A\&A, 381, 841

Hasinger, G., Giacconi, R., Gunn, J. E., et al. 1998, A\&A, 340, L27

Hasinger, G., Altieri, B., Arnaud, M., et al. 2001, A\&A, 365, L45

Holden, B., Stanford, S. A., Rosati, P., et al. 2002, Tracing Cosmic Evolution with Galaxy Clusters, ed. S. Borgani, M. Mezzetti, \& R. Valdarnini, ASP Conf. Proc., 268, 35 [astro-ph/0110032]

Jørgensen, H. E., et al. 2003, in preparation

Majerowicz, S., Neumann, D. M., \& Reiprich, T. H. 2002, A\&A, 394, 77

Martini, P., Kelson, D. D., Mulchaey, J. S., \& Trager, S. C. 2002, ApJ, 576, L109

Miyaji, T., Hasinger, G., \& Schmidt, M., 2000, A\&A, 353, 25

Neumann, D. M., \& Böhringer, H. 1997, MNRAS, 289, 123

Neumann, D. M., \& Arnaud, M. 1999, A\&A, 348, 711

Nonino, M., Bertin, E., \& da Costa, L., et al. 1999, A\&A, 137, 51

Olsen, L. F., Scodeggio, M., \& da Costa, L., et al. 1999, A\&A, 345, 681

Perrenod, S.C., 1980, ApJ, 236, 373

Pierre, M., Valtchanov, I. , \& Refregier, A. 2003, New visions of the X-ray Universe in the XMM-NEWTON and CHANDRA era, ed. F. Jansen et al. , ESA SP-488, in press, [astro-ph/0202117]

Prandoni, I., Wichmann, R., da Costa, L., et al. 1999, A\&A, 345, 448

Rosati, P., Della Ceca, R., Norman, C., \& Giacconi, R. 1998, ApJ, 492, L21

Rosati, P., Stanford, S. A., Eisenhardt, P. R., et al. 1999, AJ, 118, 76

Rosati, P., Borgani, S., \& Norman, C. 2002, ARA\&A, 40, 539

Scodeggio, M., Olsen, L. F., da Costa, L., et al. 1999, A\&AS, 137, 83

Stanford, S. A., Elston, R., Eisenhardt, P. R., et al. 1997, AJ, 114, 2232

Stanford, S. A., Holden, B., Rosati, P., et al. 2001, ApJ, 552, 504

Stanford, S. A., Holden, B., Rosati, P., et al. 2002, AJ, 123, 619 